\title{
Uptake studies in rat Peyer's patches, cytotoxicity and release studies of alginate coated chitosan nanoparticles for mucosal vaccination
}

\author{
Olga Borges ${ }^{a, *}$, Anabela Cordeiro-da-Silva ${ }^{b}$, Stefan G. Romeijn ${ }^{c}$, Maryam Amidi ${ }^{c}$, \\ Adriano de Sousa ${ }^{a}$, Gerrit Borchard ${ }^{\mathrm{c}, \mathrm{d}}$, Hans E. Junginger ${ }^{\mathrm{c}, \mathrm{e}}$ \\ ${ }^{\text {a }}$ Center for Pharmaceutical Studies, Laboratory of Pharmaceutical Technology, Faculty of Pharmacy, University of Coimbra, \\ Rua do Norte, 3000-295 Coimbra, Portugal \\ b IBMC and Laboratory of Biochemical, Faculty of Pharmacy, University of Porto, 4050-047 Porto, Portugal \\ c Leiden/Amsterdam Center for Drug Research, Division of Pharmaceutical Technology, P.O. Box 9502, 2300 RA Leiden, The Netherlands \\ d School of Pharmacy Geneva/Lausanne, University of Geneva, 1211 Geneva 4, Switzerland \\ e Faculty of Pharmaceutical Sciences, Naresuan University, Phitsanulok 65 000, Thailand
}

Received 3 March 2006; accepted 12 June 2006

Available online 16 June 2006

\begin{abstract}
The design of particulate vaccine delivery systems, particularly for mucosal surfaces, has been a focus of interest in recent years. In this context, we have previously described the development and the characterization of a new nanosized delivery system, consisting of a model antigen adsorbed to chitosan particles and coated with sodium alginate. In the present work the ovalbumin release profiles from these coated nanoparticles in different $\mathrm{pH}$ buffers were investigated and compared to those of the uncoated particles. Cytotoxicity of the polymers and nanoparticles was assessed using the MTT assay. Finally, particle uptake studies in rat Peyer's patches were performed. It was demonstrated that the coating of the nanoparticles with sodium alginate not only avoided a burst release observed with uncoated particles but also increased the stability of the particles at $\mathrm{pH} 6.8$ and 7.4 at $37{ }^{\circ} \mathrm{C}$. At neutral $\mathrm{pH}$, the release was lower than $5 \%$ after $3.5 \mathrm{~h}$ incubation in a low ionic strength buffer. For both, chitosan and alginate polymers, and for the nanoparticles, comparable cell viability data close to $100 \%$, were obtained. Additionally, based on confocal laser scanning microscopy observations, it was shown that alginate coated nanoparticles were able to be taken up by rat Peyer's patches, rendering them suitable carriers for intestinal mucosal vaccination.
\end{abstract}

(C) 2006 Elsevier B.V. All rights reserved.

Keywords: Coated nanoparticles; Chitosan; Sodium alginate; Peyer's patches; Cytotoxicity

\section{Introduction}

The primary reason for using the mucosal route of vaccination is that it is the most effective route to induce a local protective immune response, resulting in the release of $\operatorname{sIgA}$, against infections originating at a mucosal surfaces [1,2]. However, only few vaccines currently approved for human use are administered mucosally, most of which are live-attenuated ones. Mucosal vaccines, comprising soluble protein antigens, in general yielded rather disappointing results. Many factors have been described

\footnotetext{
* Corresponding author. Tel.: +351 239859927; fax: +351 239827126.

E-mail address: olga@ci.uc.pt (O. Borges).
}

causing this problem [2] and different strategies have been applied to meet this challenge $[2,3]$. One such strategy is the development of polymeric nanoparticulate delivery systems. In general, these systems are thought to promote entrapment and retention of antigens in local lymph nodes [4]. On the other hand, they might protect the antigens from the adverse environment in the presence of hydrolytic enzymes or low $\mathrm{pH}$ at the gastrointestinal mucosal surface.

In a recent publication we have described the development and the characterization of a new nanosized delivery system, consisting of chitosan nanoparticles with ovalbumin adsorbed at the surface and coated with sodium alginate [5]. These particles were designed for crossing mucosal barriers and releasing the antigen 
into lymphoid tissue, in particular in the ileal Peyer's patches. In the present manuscript, successive studies related to this new delivery system are presented.

One of the objectives of the present study was to assess the model vaccine release profiles from coated nanoparticles in order to examine the nature of the interactions between the chitosan core and the alginate coating. Furthermore, these studies were performed to obtain information about the in vivo antigen released from the particles.

The second objective was to evaluate the potential of these coated particles for being taken up by Peyer's patches. Mucosal uptake of microparticulates has been a matter of several reviews $[6,7]$. Particularly, a number of studies, not only in rodent animal models [8,9] but also in porcine gut [10] showed the uptake of chitosan nanoparticles by Peyer's patches [8,9] or by gut villous cells [10]. Likewise, studies using in vitro cell culture models demonstrated the uptake of chitosan nanoparticles by a number of different cell lines [9,11-15] and it was emphasised that the polycationic, mucoadhesive chitosan nanoparticles revealed a stronger association with mucus-secreting cells MTX-E12 than with Caco-2 cell monolayers [9]. An intense electrostatic interaction between positively charged chitosan nanoparticles and negatively charged mucins is regarded as the cause for the strong interaction of chitosan with mucus $[9,16]$. In one of our own recent studies the coating of the chitosan nanoparticles with sodium alginate has inverted the surface charge of the particles from positive to negative values [5], however, the hydrophilicity of these mucoadhesive [17] nanoparticles remained unaltered. The present coated nanoparticles presumably have the same surface properties as alginate nanoparticles and recent studies [1820] have shown that alginate particles, despite of their negative surface charge, are also able to be taken up by Peyer's patches. Thus, we examined the internalization of these coated particles by Peyer's patches.

Finally, the third objective was to evaluate the cytotoxicity of the coated and uncoated nanoparticles in comparison to the polymers used to prepare these particles. The in vitro evaluation of the cytotoxicity of chitosan has been performed in a number of previous studies [11,21-24] with different cell lines and its low cytotoxicity has already been demonstrated. However, different results were found in the literature, which may possibly be related to the presence of impurities on the different sources of bulk polymer. On the other hand, the differences observed can also be associated with the concentration of the polymer in the cell culture or to different incubation times of the polymer with the cells or finally to the cell line used. For instance for A549 cells, a cell viability near $100 \%$ (MTT assay) was demonstrated [11] with concentrations lower than $0.74 \mathrm{mg} / \mathrm{ml}$, and a mean $\mathrm{IC}_{50}$ value of $1.1 \mathrm{mg} / \mathrm{ml}$ and $1.2 \mathrm{mg} / \mathrm{ml}$ for chitosan and chitosan nanoparticles, respectively. With Calu- 3 cells a concentration of $1.5 \%$ of chitosan in culture medium decreased the cell viability to $30 \%$ [23]. Similarly, sodium alginate has been widely used because of its biodegradability, biocompatibility [25] and low toxicity [26]. Examples of its utilization include the immobilization of living cells [27] and the production of antigen delivery systems [28].

The MTT assay is considered an easy and rapid test for the evaluation of cytotoxicity and was used in this work to assess the cytotoxicity of the polymers, chitosan or sodium alginate and the alginate coated or uncoated chitosan nanoparticles in mice spleen cells.

\section{Materials and methods}

\subsection{Materials}

\subsubsection{Polymers}

Chitosan (ChitoClear ${ }^{\mathrm{TM}}$ ) was purchased from Primex BioChemicals AS (Avaldsnes, Norway). According to the provider's specifications, the degree of deacetylation was $95 \%$ (titration method) and the viscosity was $8 \mathrm{cP}$ (measured in $1 \%$ solutions in $1 \%$ acetic acid). Sodium alginate (MANUCOL LB ${ }^{\circledR}$ ) was kindly donated by ISP Technologies Inc. (Surrey, UK).

\subsubsection{Reagents}

Ovalbumin (grade V; minimum 98\%), [3-(4,5-dimethylthiazol-2-yl)] 2,5-diphenyl tetrazolium bromide (MTT), trypan blue and concanavalin A (con A) were purchased from Sigma Chemicals (St. Louis, USA), certified fetal bovine serum (fbs), Lglutamine $(200 \mathrm{mM})$ and gentamicin were from Gibco (Invitrogen Co., Paisley, Scotland, UK), 1 M HEPES buffer $(0.85 \%$ $\mathrm{NaCl}$ ) and RPMI 1640 without L-glutamine were from Biowhitaker (Cambrex Bio Science, Verviers, Belgium), ovalbumin fluorescein conjugate (3.9 mol dye/mole protein) and BODIPY ${ }^{\circledR}$ 665/676 from Molecular Probes (Leiden, The Netherlands). All other reagents used were of analytical grade. All solutions were prepared in Millipore water.

\subsection{Preparation of alginate coated chitosan nanoparticles}

Alginate coated chitosan nanoparticles were prepared by the method described previously [5]. Chitosan was dissolved at a concentration of $0.25 \%(\mathrm{w} / \mathrm{v})$ in an acetic acid solution and the formation of the particles was achieved after the addition of $3.5 \mathrm{ml}$ of sodium sulfate solution $(10 \% \mathrm{w} / \mathrm{v})$ to $200 \mathrm{ml}$ of the chitosan solution. The resultant suspension of particles was centrifuged for $30 \mathrm{~min}$ at $3500 \mathrm{rpm}(2800 \times \mathrm{g})$ and the supernatant was discarded. The particles were suspended in Millipore water and centrifuged twice more and then were frozen in liquid nitrogen and freezedried overnight using a Christ freeze-dryer (Osterode am Harz, Germany). The dry powder was kept frozen until further use.

The loading with ovalbumin was performed by incubating a solution of ovalbumin with a suspension of chitosan particles at $\mathrm{pH}$ 7.4 (phosphate buffer) under mild agitation at room temperature during $120 \mathrm{~min}$. The resulting suspension of $0.25 \%(\mathrm{w} / \mathrm{v})$ ovalbumin and $0.4 \%(\mathrm{w} / \mathrm{v})$ nanoparticles was used in successive release studies as uncoated loaded nanoparticles and was used in the subsequent coating step. Alginate coated nanoparticles were obtained by mixing of equal volumes of nanoparticles suspension and a buffer phosphate solution of sodium alginate $(1 \% \mathrm{w} / \mathrm{v})$ under magnetic stirring. The agitation was maintained during a $20 \mathrm{~min}$ period. The suspension was then centrifuged for $10 \mathrm{~min}$ at $1600 \mathrm{rpm}$ and the supernatant was discarded. The particles were re-suspended in $0.524 \mathrm{mM} \mathrm{CaCl}_{2}$ in $50 \mathrm{mM}$ HEPES buffer solution and kept under agitation for another $10 \mathrm{~min}$. 


\subsection{In vitro release studies}

Ovalbumin release from the coated and uncoated particles was performed by incubation in several buffers: $8 \mathrm{mM}$ phosphate buffer $\mathrm{pH}$ 7.4, $100 \mathrm{mM}$ phosphate buffer $\mathrm{pH}$ 5.5, 5 mM HEPES buffer $\mathrm{pH}$ 7.4, simulated intestinal fluid (SIF) and in simulated gastric fluid (SGF), as described in USP XXIV. The nanoparticle suspensions were added (1:4) to individual tubes containing the release medium previously equilibrated at $37^{\circ} \mathrm{C}$ and placed in a shaker bath adjusted to $50 \mathrm{rpm}$. At appropriate time intervals, samples were taken from each tube and filtered through a low protein-binding filter (MILLEX ${ }^{\circledR} \mathrm{GV}-0.22 \mu \mathrm{m}$; Durapore PVDF membrane; MILLIPORE, Molsheim, France) followed by centrifugation for $20 \mathrm{~min}$ at $14,000 \mathrm{rpm}$. The concentration of ovalbumin in the supernatant was assayed by BCA-protein assay. Simultaneously coated and uncoated blank (without ovalbumin) nanoparticle suspensions were subjected to the same conditions and the filtered samples were used as blanks in the BCA-protein assay. For the determination of the total protein content in the particle suspension, $0.5 \mathrm{ml}$ of the suspensions of the coated and uncoated particles were incubated with $1.5 \mathrm{ml}$ of $0.085 \mathrm{~N}$ hydrochloric acid solution $(\mathrm{pH}=1.2)$ in an ultrasound bath for $30 \mathrm{~min}$, followed by $3 \mathrm{~h}$ in a water bath at $37^{\circ} \mathrm{C}$. The samples were filtered and the protein was assayed by BCA-protein assay. Suspensions of unloaded particles were analysed under the same conditions and were used as a blank for the correction of the OD value of the samples analyzed with BCA-protein assay. In order to calculate the amount of the ovalbumin encapsulated, the concentration of unbound ovalbumin in the supernatant of particle suspensions was also determined and subtracted from the total concentration of the ovalbumin in suspension. All experiments were performed at least in triplicate.

The data of ovalbumin release from the coated nanoparticles in the phosphate buffers at different pHs $(5.5,6.8$ and 7.4), were analyzed according to zero-order kinetics, first-order kinetics, Higuchi, Korsmeyer-Peppas, Hopfenberg and Weibull as described in [29]. All kinetic models were fit to the release date (graph $M_{t} / M_{0}$ versus time) using the SigmaPlot software (version 8.0, SPSS Inc.)

The drug loading capacity (LC) and loading efficacy (LE) of the nanoparticles were calculated using the following equations:

$$
\begin{aligned}
\mathrm{LC}(\mathrm{w} / \mathrm{w})= & (\text { total amount of ovalbumin }- \text { non } \\
& - \text { bound ovalbumin }) / \text { weight of the } \\
& \text { nanoparticles }
\end{aligned}
$$

LE $(\%)=($ total amount of ovalbumin - non

- bound ovalbumin)/total amount of ovalbumin

$* 100$

\subsection{Cytotoxicity studies}

\subsubsection{Preparation of single cell suspensions of spleen cells}

Three 8-week old female BALB/c mice (Harlan Iberica, Barcelona, Spain) were sacrificed by cervical dislocation and their spleens were aseptically removed. Individual spleen cell suspensions were prepared in a Petri dish using curved needles and washed twice with RPMI 1640. The final suspension was adjusted to a final concentration of $1 \times 10^{7}$ cells per ml in complete
RPMI 1640 medium (supplemented with 10\% (v/v) fetal bovine serum, $1 \%(\mathrm{v} / \mathrm{v})$ glutamine, $1 \%(\mathrm{v} / \mathrm{v})$ gentamicin and $2 \%(\mathrm{v} / \mathrm{v})$ 1 M HEPES buffer).

\subsubsection{Solutions of the polymers}

The $0.25 \%$ chitosan test solution was prepared with complete RPMI and the $0.25 \%$ sodium alginate was prepared in $50 \mathrm{mM}$ HEPES buffer $(\mathrm{pH}=7.4)$. The solutions were heated and sonicated in order to completely dissolve the polymers. Immediately prior to incubation with the cells, the solutions were aseptically filtered.

\subsubsection{In vitro stimulation of spleen cells with polymer solutions and nanoparticle suspensions and MTT assay}

Using sterile 96-well tissue culture plates (TPP, Switzerland), $25 \mu 1$ of splenocyte suspension from three mice were plated individually, in triplicate along with $20 \mu \mathrm{l}$ of a concanavalin A solution $(50 \mu \mathrm{g} / \mathrm{ml}$ in complete RPMI solution) and $135 \mu \mathrm{l}$ of a complete RPMI solution of the polymer or suspension of the nanoparticles and incubated ( $95 \%$ relative humidity, $5 \% \mathrm{CO}_{2}$ ) for $20 \mathrm{~h}$. Assayed were $138.8 \mu \mathrm{g} / \mathrm{ml}$ and $208.3 \mu \mathrm{g} / \mathrm{ml}$ of the polymers (chitosan and sodium alginate), respectively, and $277.6 \mu \mathrm{g} / \mathrm{ml}$ and $416.6 \mu \mathrm{g} / \mathrm{ml}$ of the coated and uncoated nanoparticles, respectively.

The solvents used for the preparation of the polymer solutions (see Section 2.4.2) were also assayed as a control of the experiment. In the same way, the solutions of the polymers and the suspension of the particles were assayed without cells in order to evaluate possible interferences of the polymers or the particles in the MTT assay.

Cytotoxicity was evaluated by measuring the reduction of MTT by the mitochondrial dehydrogenase of living cells as an indication of cell viability. $18 \mu \mathrm{l}$ of MTT solution $(5 \mathrm{mg} / \mathrm{ml}$ in a phosphate buffered saline $\mathrm{pH}$ 7.4) were added to each well following $2.5 \mathrm{~h}$ of additional incubation at $37{ }^{\circ} \mathrm{C}$. To ensure solubilization of the formazan crystals, $100 \mu 1$ of $0.1 \mathrm{~N}$ isopropanol- $\mathrm{HCl}$ was added to each well and the optical density values were measured at $570 \mathrm{~nm}$ using a microplate reader. The relative cell viability (\%) related to control wells containing spleen cells in culture medium without polymer was calculated by equation:

$\%$ cell viability $=[A]$ test $/[A]$ control $\times 100$

In order to analyse and compare the results for the control group with the polymer and nanoparticle groups, a one-way ANOVA analysis followed by Dunnett's post test was performed using the GraphPad Prism 4 software. Significant differences were considered for $p<0.05$.

\subsubsection{Trypan blue and propidium iodide stain}

The viability of the splenocytes was also evaluated by the capability of these cells with uncompromised membrane integrity to exclude the dye. The in vitro stimulation of the cells with the polymers or the nanoparticles was done as described above in the absence of concanavalin A and for a period of $15 \mathrm{~h}$. Three samples of the same animal were mixed with a PBS solution of trypan blue and the resultant suspensions ( $0.2 \%$ trypan blue) were visualized using a standard light microscope. Stained and unstained cells were counted manually using a hemocytometer. Additionally, the pooled culture cells from the same animal and the same 
formulation test were also stained with propidium iodide (PI) $(0.25 \mu \mathrm{g} / \mathrm{ml})$ and the fluorescence immediately measured with a fluorescence activated cell sorter (FACS Calibur) (BD, Biosciences, Madrid, Spain). Data were analysed by Cell Quest software (BD, Biosciences, Madrid, Spain).

Data were presented as means \pm S.E.M. for three experiments and statistical significance was assessed using repeated measured one-way ANOVA followed by Dunnett's post test using the GraphPad Prism 4 software. Significant differences were considered for $p<0.05$.

\subsection{Uptake studies in rat Peyer's patches}

The studies with Wistar rats (Charles River, Someren, The Netherlands) were performed according to the guidelines of the Ethical Committee of Leiden University. Male rats weighing 250-340 g were housed for acclimatization one week before the experiments with free access to food and water with $12 \mathrm{~h}$ light/ dark cycle. On the day before the experiment they were starved overnight, allowing only free access to water.

The rats were anaesthetized by IM administration of $0.5 \mathrm{ml} / \mathrm{kg}$ of Hypnorm ${ }^{\circledR}$ (fentanyl citrate $0.315 \mathrm{mg} / \mathrm{ml}$ and fluanisone $10 \mathrm{mg}$ ) $\mathrm{ml}$ ) and $0.5 \mathrm{ml} / \mathrm{kg}$ of Dormicum ${ }^{\circledR}$ (midazolam $5 \mathrm{mg} / \mathrm{ml}$ ). The animals remained anaesthetized throughout the experiment and were placed on electrical heating mats. A small incision in the lower stomach was made and a teflon tube $(\varnothing: 0.5 \mathrm{~mm}$ I.D. $\times 1.0 \mathrm{~mm}$ O. D.) was introduced through the pylorus approximately 3 to $5 \mathrm{~cm}$ into the duodenum. The coated particle suspensions loaded with FITC-ovalbumin or the $0.05 \%$ FITC-ovalbumin PBS solutions were administered $(500 \mu \mathrm{l})$ and the incision was closed after the removal of the tube from the stomach. The rats were sacrificed after $2 \mathrm{~h}$ by cervical dislocation. The whole intestine was removed and flushed with $20 \mathrm{ml}$ of phosphate buffered saline. Between 4 and 5 Peyer's patches were excised from each intestine and fixed with $2 \%$ paraformaldehyde, rinsed again with $\operatorname{PBS}\left(4^{\circ} \mathrm{C}\right)$ and the tissue was permeabilized by immersion in $0.1 \%$ Triton X-100 (PBS) for $20 \mathrm{~min}$. The tissue was rinsed again and stained with a $0.0617 \%$ solution of BODIPY ${ }^{\circledR}$ 665/676 (Pierce, Leiden, The Netherlands) in methanol for $60 \mathrm{~min}$. Finally, the Peyer's patches were mounted on glass slides and observed using a confocal laser scanning microscope (CLSM) (Bio-Rad, Alphen a/d Rijn, The Netherlands). The confocal images were obtained by scanning the tissue samples in the $x, y$ plane with a $z$-step of $500 \mathrm{~nm}$.

Fluorescein label (FITC) coated nanoparticles were prepared according to the procedure described above, using a $0.05 \%$ FITC-ovalbumin solution in phosphate buffered saline.

Three rats for the group control (solution of the FITCovalbumin) and four rats for the group treated with the suspension of the FITC-coated nanoparticles were included in this study.

\section{Results and discussion}

\subsection{Characterization of the nanoparticles}

In a recent publication [5] of our group, the development of alginate coated chitosan nanoparticles was described and the characterization of this new delivery system reported. Before coating with sodium alginate, chitosan nanoparticles had a mean diameter of $643 \mathrm{~nm}$ (dynamic light scattering technique) and were positively charged $(+37 \mathrm{mV})$. Scanning electron microscopy (SEM) images of uncoated particles also revealed the presence of small nanoparticles (around $100 \mathrm{~nm}$ ). After the coating process, however, SEM micrographs allowed us to verify that most of the particles were in a range between 300 and $600 \mathrm{~nm}$. The presence of the alginate coating layer was confirmed by FTIR and DSC studies and by the observation of the inversion of the zeta potential $(-34 \mathrm{mV})$ of coated nanoparticles. The loading of the nanoparticles was done by an adsorption process based on electrostatic interaction [30-32] between the negatively charged ovalbumin at pH 7.4 and the positively charged chitosan nanoparticles. The excellent properties of the chitosan delivery systems to adsorb at its surface proteins [33,34] and vaccines [30,35], renders them a promising carrier systems. In the present work, the ovalbumin loading efficacy (see Eq. (2)) of uncoated particles prepared under the conditions described before was $75 \%$, and the loading capacity (see Eq. (1)) was $0.49 \mathrm{mg}$ ovalbumin/mg of nanoparticles which are close to those results found in literature [34]. Smaller values of those quantities, of loading efficacy and loading capacity, 57\% and $0.39 \mathrm{mg}$ ovalb./mg particles respectively, were observed for the alginate coated particles.

Moreover, physical degradation, including aggregation of the ovalbumin, released from the coated nanoparticles, was not observed as was previously demonstrated by SDS-PAGE [5]. This result was not a surprise because the ovalbumin loading process, as well as the following step, the coating of the nanoparticles with sodium alginate, were performed using a phosphate buffer ( $\mathrm{pH}$ 7.4), considered as mild entrapment process, having no deleterious effect on the conformation of the protein [36].

\subsection{In vitro release studies}

\subsubsection{Ovalbumin release profiles from chitosan nanoparticles}

In vitro release studies in various aqueous media were performed in order to evaluate the differences of ovalbumin release profiles from uncoated and coated nanoparticles and as an attempt to understand the physicochemical structure of these two delivery systems. The results are illustrated in Fig. 1 for the uncoated chitosan nanoparticles and in Fig. 2 for the alginate coated chitosan nanoparticles.

An almost complete ovalbumin desorption from uncoated particles was observed immediately after the addition of the suspension of the particles to the equilibrated release medium at $\mathrm{pH} 1.2$ (simulated gastric fluid) and $\mathrm{pH} 5.5$ (100 mM buffer phosphate). At low $\mathrm{pH}$ values, chitosan nanoparticles displayed probably the highest positive surface charge due to the protonation of the free amine groups of chitosan. Additionally, ovalbumin has also predominantly positive charges below its isoelectric point (ovalbumin isoforms: $\mathrm{p} I$ between 4.8 and 5.0) [37], resulting from the protonation of the amine group. The combination of these two factors can explain the immediate desorption of ovalbumin. Such behaviour, the burst release, also allowed us to suggest that ovalbumin was mostly adsorbed to the surface of the particles and not inside the pores. 


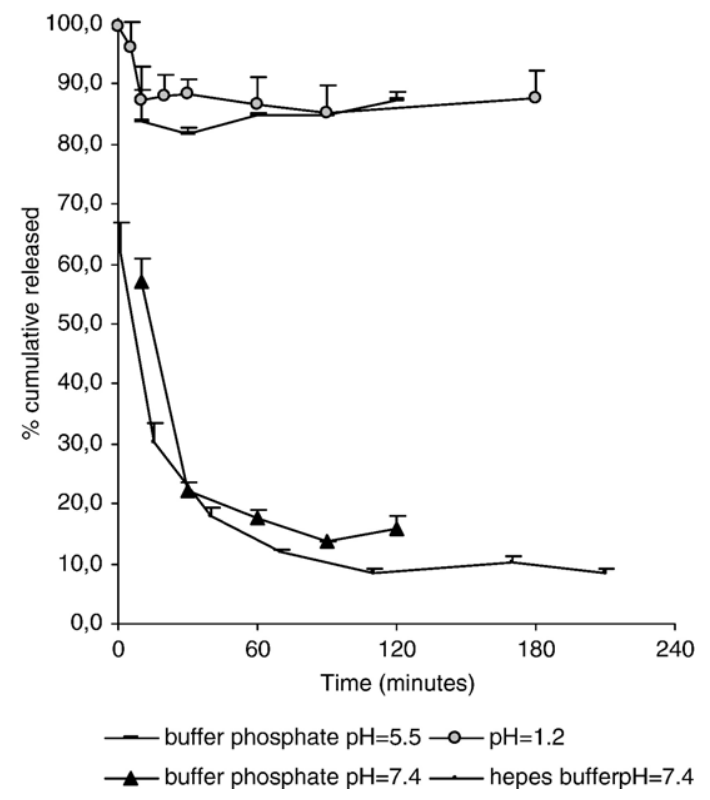

Fig. 1. In vitro release profiles of ovalbumin from uncoated chitosan nanoparticles into various aqueous dissolution media at $37^{\circ} \mathrm{C}$. Mean \pm standard deviation, $n=3$.

On the other hand, when the release studies of uncoated particles were conducted at $\mathrm{pH} 7.4$ (phosphate buffer), the initial white colour of the nanoparticle suspension disappeared, immediately after its addition to the release medium, reappearing, in a progressive way after a few seconds. This phenomenon was believed to be related to the destruction of the initial structure of the nanoparticles as a consequence of its instability at $37{ }^{\circ} \mathrm{C}$ in phosphate buffer ( $\mathrm{pH} 7.4$ ), followed by the formation of new nanoparticles (precipitates), indicated by turbidity [32] examined by transmission measurements at $500 \mathrm{~nm}$ (data non shown). As a consequence, in the first moment after the addition of the particles suspension to the release medium, an immediate desorption of the ovalbumin from the particles occurred, to which followed a progressive re-association of the protein to the recently formed particles and the establishment of a new adsorption equilibrium. This new equilibrium was then stable, under the given conditions of buffer strength and $\mathrm{pH}$. This occurrence was reflected by the atypical release profile shown in Fig. 1. Similar results were observed when the study was repeated at $37{ }^{\circ} \mathrm{C}$ with HEPES buffer ( $\mathrm{pH}$ 7.4) (Fig. 1). Once the ovalbumin loading of the particles was conducted with the same buffer (phosphate buffer) at room temperature, it was assumed that the observed intermediate instability of the particles was probably related, not only to the $\mathrm{pH}$ of the release medium, but also to the temperature. Furthermore, the small size of the particles is also an important factor contributing to the instability of the delivery system and consequently to the initial fast release [38] of ovalbumin. This instability was also described in our own previous studies [5] in simulated intestinal fluid (SIF). However, this behaviour was not observed by others which could mean that despite of the apparently similar formulation and preparation procedures, a diversity of characteristics of the chitosan particles exist [33,34,39-43]. The cause of different release behaviours from chitosan nanoparticles may be related with its process of preparation. Recently, the importance of controlling the $\mathrm{pH}$ of the formulation during chitosan nanoparticles preparation was demonstrated [14]. Other parameters, such as deacetylation degree and molecular weight of the polymer also has an impact on the properties of the final nanoparticles [33]. Nevertheless, it should be emphasised that a correct procedure during the release studies must be followed, including the rigorous verification of the temperature of the release medium, $37^{\circ} \mathrm{C}$, before the start of the experiment.

\subsubsection{Ovalbumin release profiles from alginate coated chitosan nanoparticles}

In contrast to uncoated chitosan nanoparticles, the release profile of ovalbumin from nanoparticles was significantly modified after coating with sodium alginate (Fig. 2a). In a pH 7.4 (phosphate buffer) more than $40 \%$ of the ovalbumin was released within the first $60 \mathrm{~min}$ and during the following $16 \mathrm{~h}$ not more than an additional 25\% was released. On the other hand, when using HEPES buffer of the same $\mathrm{pH}$ or plain water, not more than $5.8 \%$ of the ovalbumin was released within the first $3 \mathrm{~h}$ and only $4 \%$ of ovalbumin was released in water after $17.5 \mathrm{~h}$ from the alginate-coated particles. The observed differences at the same $\mathrm{pH}$, are believed to be related, not only to the ionic strength of the buffers used, but also to the composition of the buffer. The high affinity of phosphate ions to the calcium ions is well known. Therefore, when $\mathrm{pH}$ phosphate buffers are used, the destabilizing of calcium-crosslinked alginate matrix has to be taken into account. This effect may cause a faster opening or breakdown of crosslinking compared to the results in HEPES buffer or to plain water where this reaction does not occur. The loss of the calcium ions from the alginate coating, has as a consequence, the increase of the permeability of the coating and consequently an increased diffusion of the ovalbumin into the release medium. Furthermore, the highest ionic strength of the phosphate buffer, compared with HEPES buffer and water should have an important impact on the weakening of the interactions, between the chitosan core and the alginate coating. This dependence on the ionic strength $(\mathrm{pH} 7.4)$ is typical of electrostatic interaction, and consequently these observations reinforce our previous considerations about the electrostatic nature of the interactions between the alginate coating and the chitosan core [5].

Finally, when the ovalbumin release study was conducted using a $\mathrm{pH} 5.5$ phosphate buffer, the differences observed when compared to the same phosphate buffer of $\mathrm{pH} 7.4$, should be attributed to the $\mathrm{pH}$ of the buffer. The $\mathrm{pH}$ (5.5) seemed to be favourable to maintain strong interactions between alginate and chitosan and this was probably the cause for the maintenance of the entrapment of ovalbumin at an elevated percentage. A comparable result was observed (Fig. 2a) at pH 6.8 in SIF.

As evident from what was referred before, the release of ovalbumin from the coated particles is actually a result of a combination of different processes which made the application of a kinetic model a difficult task [38]. In particular, the electrostatic interactions between the chitosan core and the ovalbumin should have a determinant role in the release mechanism and should be impeditive to observe a good fit with a diffusion-controlled release mechanism. In fact among several models referred, including the ones of Higuchi, Korsmeyer-Peppas, Hopfenberg 


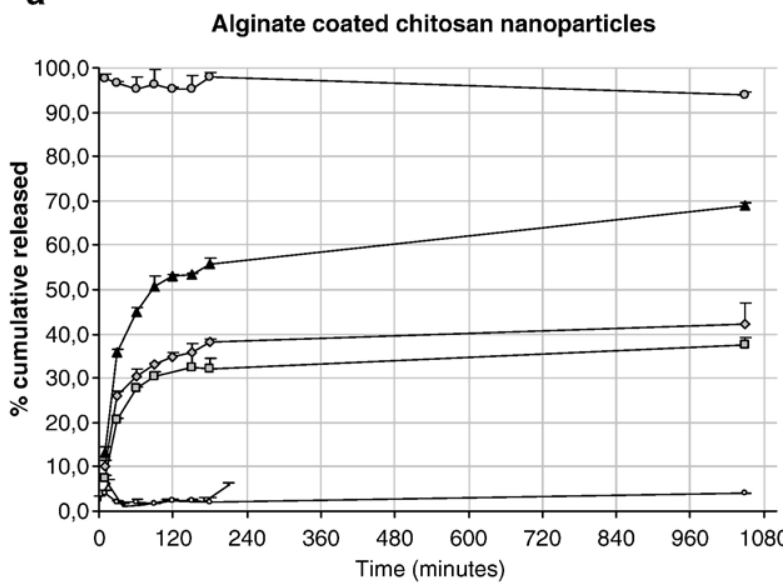

$\multimap-\mathrm{pH}=1.2 \quad \multimap$ buffer phosphate $\mathrm{pH}=5.5 \rightarrow$ buffer phosphate $\mathrm{pH}=6.8$
$\neg$ buffer phosphate $\mathrm{pH}=7.4 \longrightarrow$ hepes buffer $\mathrm{pH}=7.4 \quad \longrightarrow$ water

C

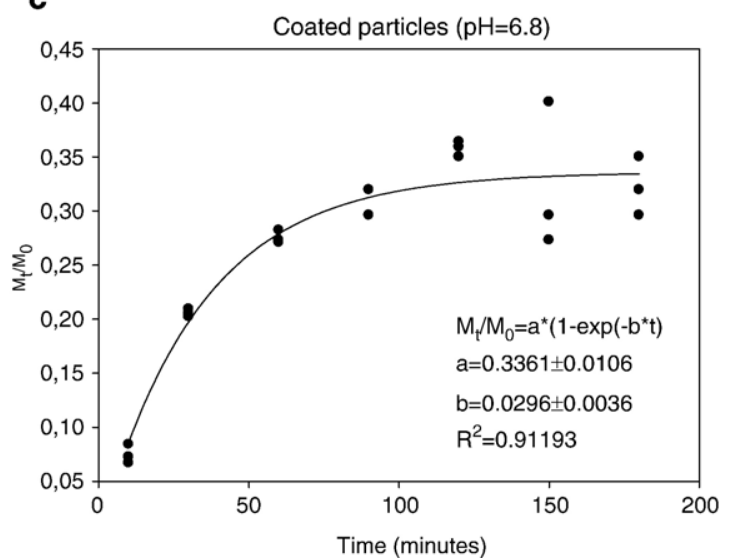

b

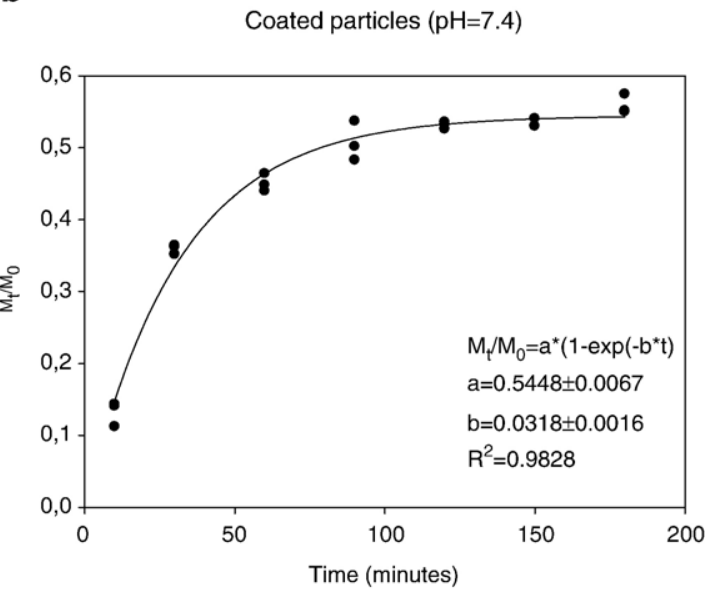

d

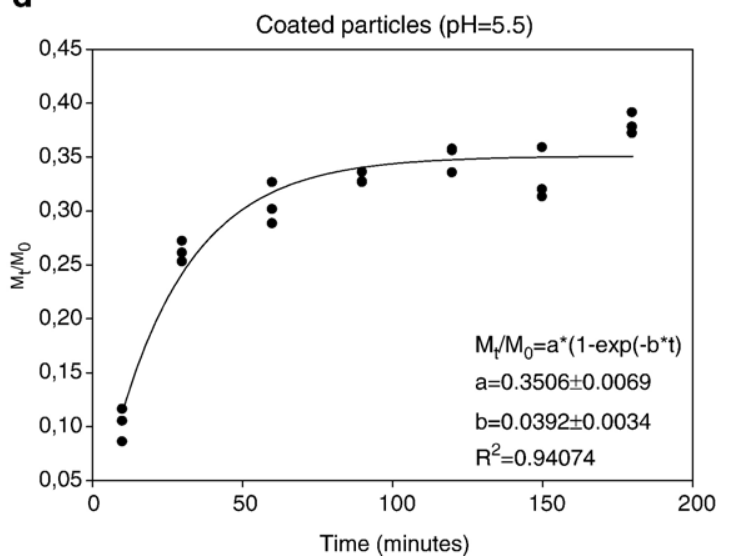

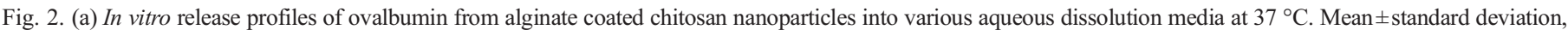

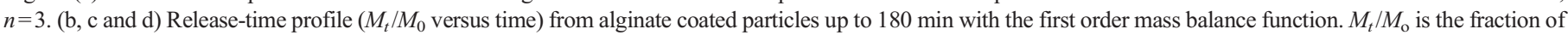
drug release after time $t$.

and Weibull [29], the $M_{t} / M_{0}$ versus time graph, from the release studies performed in the phosphate buffers at different $\mathrm{pHs}$ (5.5, 6.8 and 7.4), showed to have the best fit for the first order model (Fig. 2b,c,d), described by following equation:

$M_{t} / M_{0}=a^{*}[1-\exp (-b t)]$

Where $M_{t} / M_{0}$ is the fraction of drug released at time $t$. The two parameters of Eq. (4), the pre-exponential term a and the first-order constant $b$ were estimated by this fitting process and their values are shown in Fig. 2b,c,d. The first-order model release mechanism has been associated with particular characteristics of the delivery system; they reflected to some extent a reservoir-type delivery system [44]. Moreover, it was suggested that the first order kinetics could describe the release profile, from the pharmaceutical dosage forms such as those containing water-soluble drugs (that is the case of ovalbumin in the present study), where drugs would be released at the rates proportional to the amounts of drug remaining inside the dosage form [45].

Similar to our results, it was demonstrated in a recent study [46], that the 5-Flu release data, from both liposomes and PLGA or PLA microspheres, were modelled as a first-order process, with excellent reproducibility of the release kinetics.

The alginate coating of the nanoparticles not only improved the stability of the chitosan particles at $37^{\circ} \mathrm{C}$ in 6.8 and $7.4 \mathrm{pH}$ buffers, but also allowed a significant reduction of the ovalbumin burst release observed for the uncoated particles with the different buffers, with an exception at $\mathrm{pH} 1.2$ (SGF). The release characteristics of the present nanoparticles may be altered by the increase in complexity (presence of surfactants, enzymes, salts) of the selected release buffer. Thus in order to obtain a better knowledge of the situation in vivo, more complete in vitro models would be required.

\subsection{In vitro cell viability studies}

Splenocytes have been used for the evaluation of the toxicological profiles of several compounds and delivery systems. For example, in a recent study, the evaluation of the cytotoxicity of tetanus toxoid loaded poly(lactide-co-glycolide) nanospheres was done with splenocytes [47]. The principal reason for the choice of spleen cells in this study, was related to the fact that they are obtained and cultured easier, compared to other lymphoid organs, 
like Peyer's patches, and represent a very good and sensitive representative of the different cells of mucosal immune system. The spleen is a lymphoid organ, bridging the innate and adaptive immune system in a uniquely organized way (reviewed in Ref. [48]). A primary culture of mixed spleen cells normally contains (FACS analysis performed in our own lab) more than $50 \%$ of B lymphocytes, about $30 \%$ of T lymphocytes $(18 \% \mathrm{CD} 4+; 12 \%$ CD $8+$ ) and less than $20 \%$ erythrocytes, fibroblast, macrophages, dendritic cells, and granulocytes. Similar to the spleen, Peyer's patches also contain a large number of $\mathrm{B}$ cells, $\mathrm{T}$ cells, macrophages and dendritic cells [15]. Thus, a primary culture of spleen cells should have the requisites to mimic possible toxic effects of the nanoparticles, when the nanoparticles, internalized by Peyer's patches, remain in a close contact with those immune cells during a certain period of time.

In this study, the viability of primary culture of splenocytes in the presence of the polymers sodium alginate and chitosan, at two different concentrations, was evaluated by different methods, and in order to estimate whether the preparation process of the particles would introduce any cytotoxicity, uncoated and alginate coated chitosan particles were also studied.

The MTT test was used to evaluate the effects of the polymers and the particles on the metabolic activity of mice spleen cells. No evidence of cytotoxicity was observed for the polymer solutions (Fig. 3) or the particle suspensions (Fig. 4) and a cell viability of around $100 \%$ was observed in all test groups. Moreover, the statistical analysis of the results underlined the higher mean values of cell viability (\% of control) in the groups treated with higher concentrations of polymers, chitosan or alginate and with particles coated or uncoated (in both concentrations) when compared with the control group. Considering that proliferating cells are metabolically more active than non-proliferating (resting) cells, these results can also be interpreted as the possible impact of polymers and particles on cell proliferation. This is probably an indication that the polymers may favourably influence lysosomal

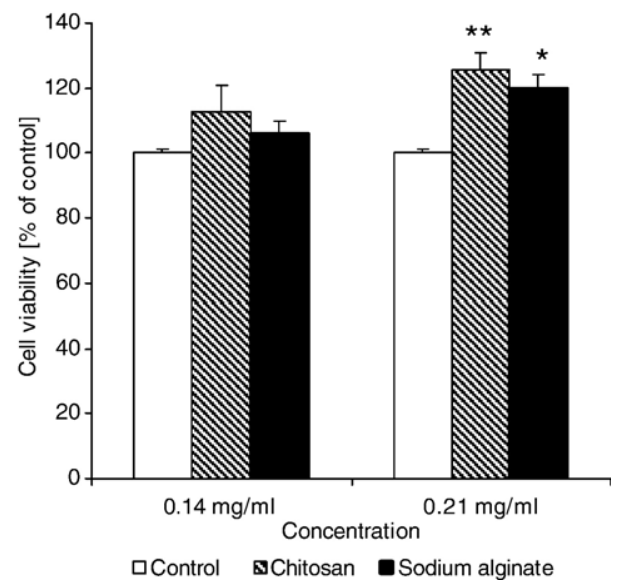

Fig. 3. Viability of splenocytes measured by the reduction of MTT. The cells were incubated for $20 \mathrm{~h}$ in the presence of two different concentrations of polymers, chitosan and sodium alginate. The control group corresponds to the assay just with cells in culture medium. Results represent the mean \pm standard error of the mean for 3 different mice, each performed in triplicate $(n=9)$. Statistical differences between control group and formulations are reported as: $* p<0.05, * *_{p}<0.01$. Cell viability $(\%$ of the control $)=[A]$ test $/[A]$ control $\times 100$.

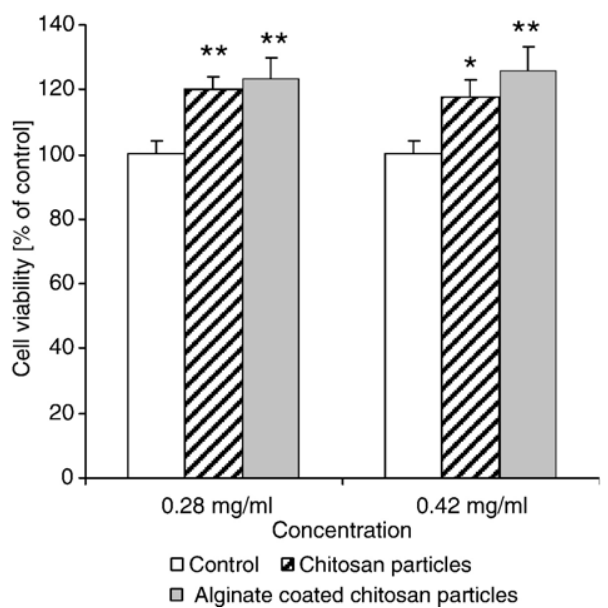

Fig. 4. Viability of splenocytes measured by the reduction of MTT. The cells were incubated for $20 \mathrm{~h}$ in the presence of two different concentrations of the nanoparticles, alginate coated chitosan particles and uncoated chitosan particles. The control group corresponds to the assay just with cells in culture medium. Results represent the mean \pm standard error of the mean for 3 different mice, each perform in triplicate $(n=9)$. Statistical difference between control group and formulations are reported as: ${ }^{*} p<0.05,{ }^{*} p<0.01$. Cell viability $(\%$ of the control $)=[A]$ test $/[A]$ control $\times 100$

and mitochondrial activity of the cells [22], nevertheless, this observation needs to be confirmed by specific experiments.

On the other hand, the cytotoxicity results as measured by the reduction of the MTT substrate could, in theory, be "masked" by cellular proliferation. Therefore, it was decided to conduct cytotoxicity studies with a different methodology using trypan blue or propidium iodide (PI) dyes for staining the cells whose membrane integrity may have been compromised. The results of this study are summarized in Figs. 5, 6, and 7. The count of nonviable splenocytes stained with trypan blue (Fig. 5) in comparison with the non-stained cells revealed that the presence of the polymers during $15 \mathrm{~h}$ did not decrease the percentage of viable cells and are in close agreement with the results obtained from the MTT assay. FACS sorting and analysis (Fig. 6) of lymphocytes previously treated with PI strengthened these observations and the proportion of dead (M1) cells in all the samples was always lower than 3\%. The statistical analysis of these results showed that the values obtained in each treatment group were not different from the control group. However, a more pronounced difference between the location of FL3 histograms from control groups (Fig. 7, left and right picture) and groups treated with a solution of the polymers chitosan or sodium alginate (Fig. 7, right picture) was observed. These results possibly indicate that in the presence of the polymers, a slight perturbation of the plasma membrane occurred and these lymphocytes are more permeable to the fluorescent nuclear probe, PI. Nevertheless, this event did not induce cell death, as was demonstrated by trypan blue dye exclusion and MTT assays. The present results correlate with those found in the literature [21] where it was demonstrated that chitosan increased the Caco-2 cell permeability in a reversible and dose-dependent way and was not accompanied by cell extrusion.

The combination of these three different methods adds reliability to the final evaluation of the cytotoxicity profiles of these polymers and nanoparticulate formulations. 


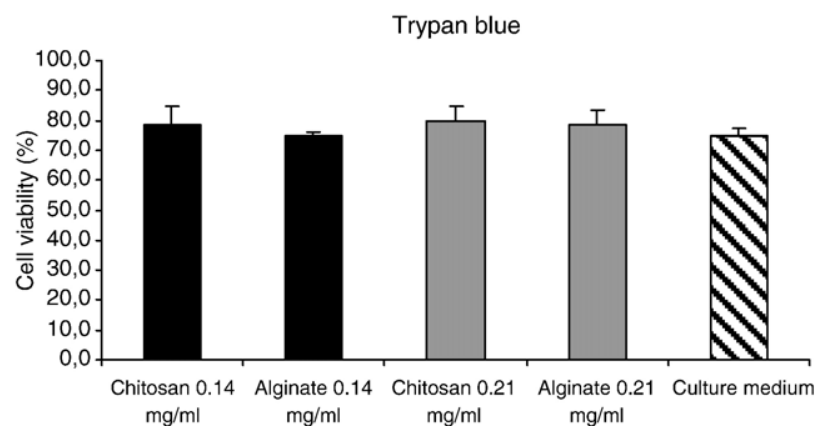

Fig. 5. The viability of splenocytes measured by counting trypan blue stained and unstained cells with hemocytometer. The cells were incubated for $15 \mathrm{~h}$ in the presence of two different concentrations of the polymers, chitosan and sodium alginate. The control group corresponds to the assay just with cells in culture medium. Results represent the mean \pm standard error of the mean for 3 different mice $(n=3)$ and each count was the result of pooled samples from the same mice. No statistical differences were found between the control and the polymer groups.

\subsection{Uptake studies of alginate coated chitosan nanoparticles into rat Peyer's patches after intra-duodenal administration}

The uptake of alginate coated chitosan nanoparticles into the rat Peyer's patches through M-cells was investigated. Chitosan particles were loaded with ovalbumin labelled with fluorescein as a model vaccine and then coated with sodium alginate. To better visualise the contour of the cells, the Peyer's patches were stained with Bodipy ${ }^{\circledR}$, which is a nonpolar lipophilic dye used to stain the cell membrane. The same methodology was recently used successfully to visualise the uptake of $N$-trimethyl chitosan nanoparticles by nasal epithelia and NALT cells [49] or to visualize chitosan nanoparticles within Peyer's patches [8]. In the present work, CLSM images of Peyer's patches from the rats treated with the suspension of fluorescent coated nanoparticles, showed the presence of fluorescent nanoparticles (green spots) inside the Peyer's patch (upper pictures in Fig. 8). The nanoparticles were visualized, not only in the region underneath $(0.5 \mu \mathrm{m})$ the follicleassociated epithelium (FAE) (right upper picture in Fig. 8), the subepithelial dome region (SED), but also in deeper regions of the secondary lymphoid organ (left upper picture in Fig. 8). Z-scan images ( 21 images), in successive steps $(0.5 \mu \mathrm{m}$ distance between steps) and starting $67 \mu \mathrm{m}$ deep from the surface of the Peyer patches, also indicated that the nanoparticles were also transported to deeper regions (data not shown). On the contrary, the photos from the control group (lower pictures in Fig. 8), treated with a solution of ovalbumin-FITC did not show any green fluorescence except a slight shade green (lower left picture) that could mean that a small amount of ovalbumin-FITC could be absorbed by the tissue.

Some of the green dots visualized in the Peyer's patches seemed not to be individual particles, but agglomerates of the nanoparticles taken up into lysosomal compartments. Focusing of sections at high power suggested that microparticle clusters were localised intracellularly (left upper picture in Fig. 8), an observation consistent with a recently published study [50] in which CLSM images were presented, showing clusters of polystyrene nanoparticles present in the subepithelial dome region underlying the Peyer's patch. Moreover, in the same study, dendritic cells labelled by immunostaining were shown to efficiently phagocytose microparticles and when the microparticles were associated to enterotoxin adjuvants, they were transported from the SED region into underlying B-cell follicles and adjacent parafollicular T-cell zones.

The SED region underlying the FAE are rich in phagocytic cells (dendritic cells and macrophages) [50]. Recent published studies, showed that alginate nanoparticles are able to be taken up not only in vivo by Peyer's patches [18-20], but also in vitro by phagocytic cells $[19,51]$. Following this idea, alginate coated chitosan nanoparticles were most likely internalized by phagocytic cells, most probably dendritic cells, present in Peyer's patches. In future work it will be important to determine the phenotype and maturation state of the microparticle-loaded cells as it can be important to follow and to know the mechanism of phagocytic cells migration.

As an important finding, this study provided an answer to our initial question related to the capability of this new delivery system, formed by hydrophilic negatively charged particles, to be internalized by Peyer's patches. In spite of the argument that
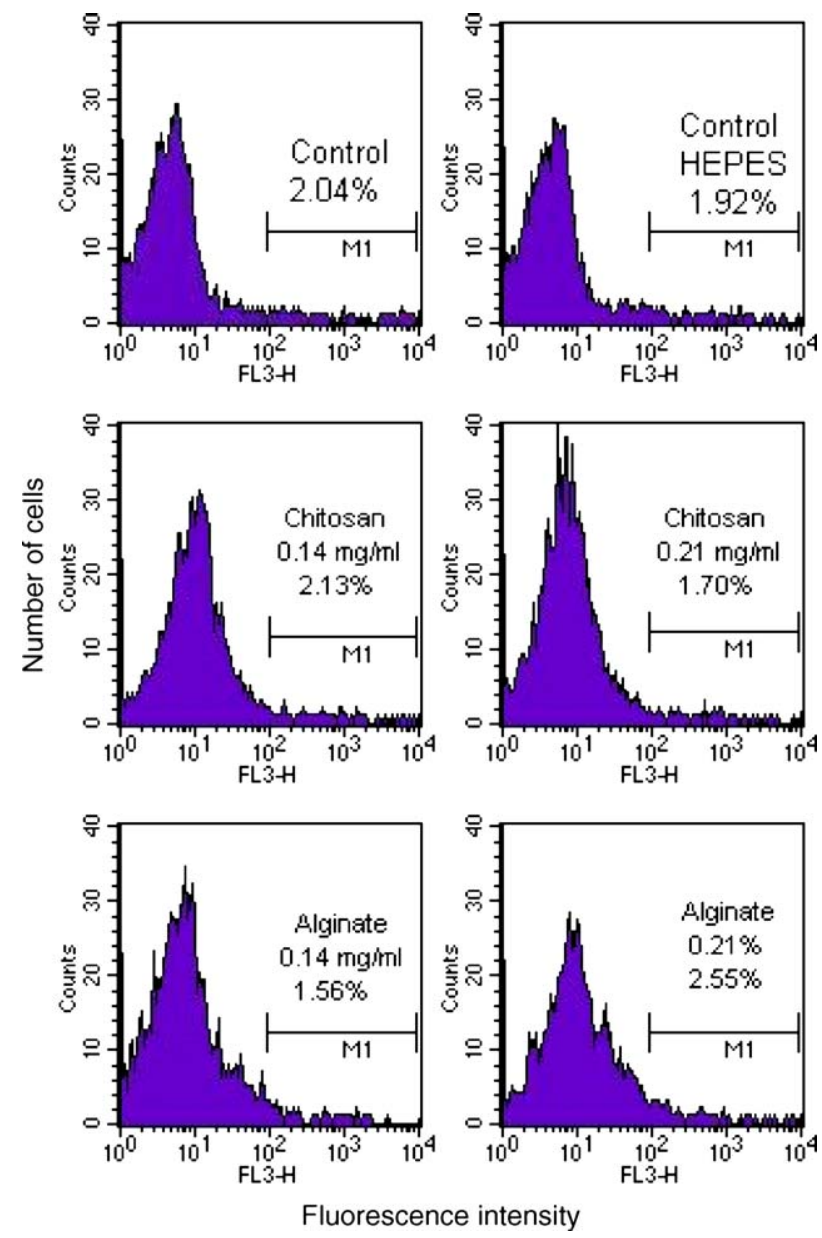

Fig. 6. Flow-cytometric analysis of mice splenocytes previously incubated in the presence of two different concentrations of the polymers chitosan and sodium alginate during $15 \mathrm{~h}$. The FL3 histograms show the proportion of PI stained nonviable (M1) and viable cells from pooled samples of one mouse. Each histogram is representative of three animals studied individually. No statistical significant differences were found when the control group was compared with the other treatment groups 


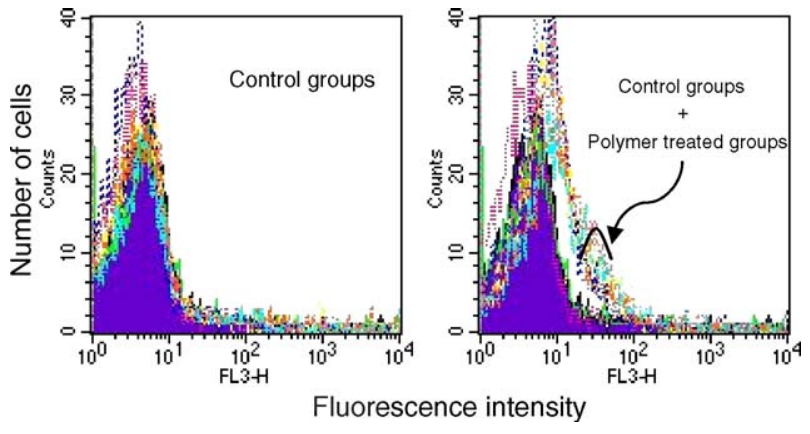

Fig. 7. Flow-cytometric FL3 histogram overlay of control groups on the left picture and control plus polymer treatment (sodium alginate and chitosan) on the right picture.

positive or a more hydrophobic surface increases cellular uptake due to its better interaction with lipophilic cell membranes, this study showed that also negatively charged hydrophilic particles are being internalised. Moreover in a recent study [51], the increase of the hydrophobicity of alginate particle surface did not improve its uptake by mouse macrophages and consequently, cellular uptake seems to be the result of a complex combination of size, hydrophobicity, as well as specific interactions between surface functional groups of alginate microspheres and the cell membrane.

\section{Conclusions}

The adsorption of therapeutic proteins and model antigens onto chitosan nanoparticles has proven to be a very mild process resulting in a very high loading efficacy. In this study, it was shown that coating of this delivery system with sodium alginate yielded coated particles, in the nanosize range, with a much better stability and controlled release properties for vaccine delivery, than the chitosan loaded cores themselves. It was also demonstrated that the preparation process of the nanoparticles did not introduce any toxic compound on the particles. On the contrary, a slight stimulation of the splenocytes co-cultured with the nanoparticles was observed. Finally it was shown that these hydrophilic coated nanoparticles even with a negative surface charge were taken up by rat Peyer's patches, which made them promising carriers for mucosal vaccination. In vivo studies with a real vaccine are underway to investigate the efficacy of this new mucosal delivery system.
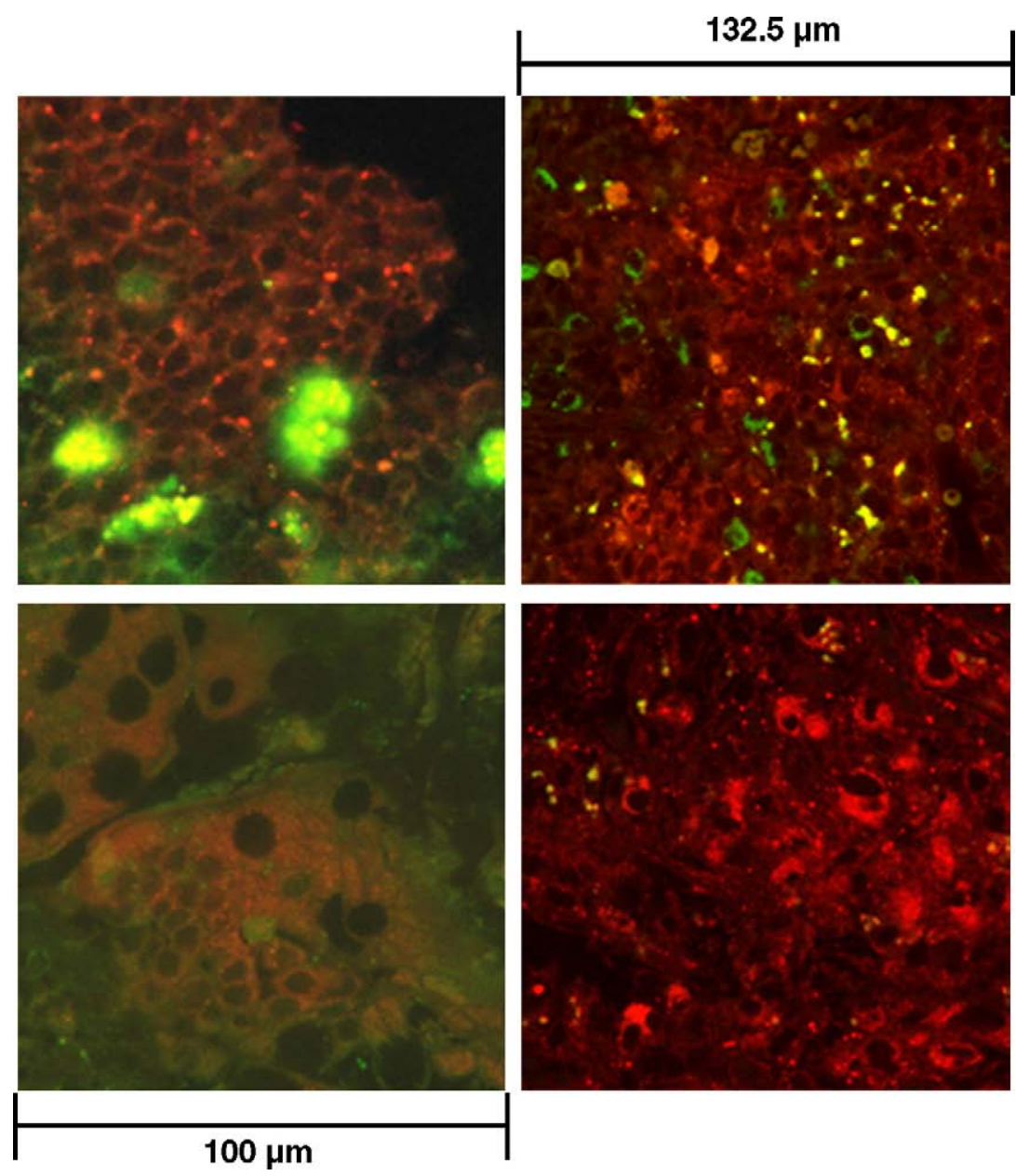

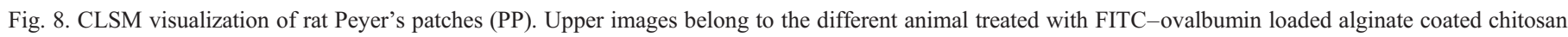

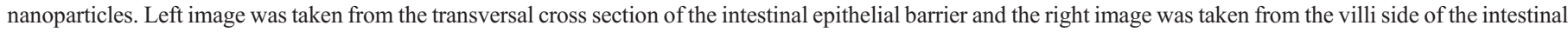

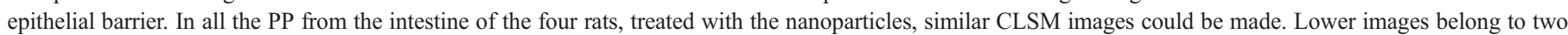

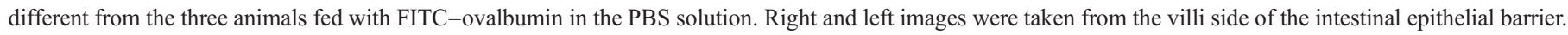




\section{Acknowledgements}

This work was supported in part by a grant from the Portuguese Ministry of Science and Technology (SFRH/BD/5327/2001).

We would like to express our thanks to H. de Bont (Division of Toxicology, Leiden/Amsterdam Center for Drug Research (LACDR), Leiden University) for his technical assistance in performing CLSM experiments.

\section{References}

[1] J. Holmgren, A. Harandi, M. Lebens, J.-B. Sun, F. Anjuère, C. Czerkinsky, Mucosal adjuvants based on cholera toxin and E. coli heat-labile enterotoxin, in: V. Schijns, D. O'Hagan (Eds.), Immunopotentiators in Modern Vaccines, Academic Press, 2006, pp. 235-252.

[2] W.S. Shalaby, Development of oral vaccines to stimulate mucosal and systemic immunity: barriers and novel strategies, Clin. Immunol. Immunopathol. 74 (1995) 127-134.

[3] J. Holmgren, C. Czerkinsky, K. Eriksson, A. Mharandi, Mucosal immunisation and adjuvants: a brief overview of recent advances and challenges, Vaccine 21 (Suppl 2) (2003) S89-S95.

[4] D. O'Hagan, Microparticles as vaccine delivery systems, in: V. Schijns, D. O’Hagan (Eds.), Immunopotentiators in Modern Vaccines, Academic Press, 2006, pp. 123-147.

[5] O. Borges, G. Borchard, J.C. Verhoef, A. de Sousa, H.E. Junginger, Preparation of coated nanoparticles for a new mucosal vaccine delivery system, Int. J. Pharm. 299 (2005) 155-166.

[6] N. Hussain, V. Jaitley, A.T. Florence, Recent advances in the understanding of uptake of microparticulates across the gastrointestinal lymphatics, Adv. Drug Deliv. Rev. 50 (2001) 107-142.

[7] T. Jung, W. Kamm, A. Breitenbach, E. Kaiserling, J.X. Xiao, T. Kissel, Biodegradable nanoparticles for oral delivery of peptides: is there a role for polymers to affect mucosal uptake? Eur. J. Pharm. Biopharm. 50 (2000) $147-160$.

[8] I.M. Van Der Lubben, F.A. Konings, G. Borchard, J.C. Verhoef, H.E. Junginger, In vivo uptake of chitosan microparticles by murine Peyer's patches: visualization studies using confocal laser scanning microscopy and immunohistochemistry, J. Drug Target. 9 (2001) 39-47.

[9] I. Behrens, A.I. Pena, M.J. Alonso, T. Kissel, Comparative uptake studies of bioadhesive and non-bioadhesive nanoparticles in human intestinal cell lines and rats: the effect of mucus on particle adsorption and transport, Pharm. Res. 19 (2002) 1185-1193.

[10] F.C. Mooren, A. Berthold, W. Domschke, J. Kreuter, Influence of chitosan microspheres on the transport of prednisolone sodium phosphate across HT29 cell monolayers, Pharm. Res. 15 (1998) 58-65.

[11] M. Huang, E. Khor, L.Y. Lim, Uptake and cytotoxicity of chitosan molecules and nanoparticles: effects of molecular weight and degree of deacetylation, Pharm. Res. 21 (2004) 344-353.

[12] T. Ishii, Y. Okahata, T. Sato, Mechanism of cell transfection with plasmid/ chitosan complexes, Biochim. Biophys. Acta 1514 (2001) 51-64.

[13] M. Huang, Z. Ma, E. Khor, L.Y. Lim, Uptake of FITC-chitosan nanoparticles by A549 cells, Pharm. Res. 19 (2002) 1488-1494.

[14] Z. Ma, H.H. Yeoh, L.Y. Lim, Formulation pH modulates the interaction of insulin with chitosan nanoparticles, J. Pharm. Sci. 91 (2002) 1396-1404.

[15] K.A. Janes, M.P. Fresneau, A. Marazuela, A. Fabra, M.J. Alonso, Chitosan nanoparticles as delivery systems for doxorubicin, J. Control. Release 73 (2001) 255-267.

[16] S.E. Harding, Mucoadhesive interactions, Biochem. Soc. Trans. 31 (2003) 1036-1041.

[17] A. Bernkop-Schnurch, C.E. Kast, M.F. Richter, Improvement in the mucoadhesive properties of alginate by the covalent attachment of cysteine, J. Control. Release 71 (2001) 277-285.

[18] V. Gerdts, R.R. Uwiera, G.K. Mutwiri, D.J. Wilson, T. Bowersock, A. Kidane, L.A. Babiuk, P.J. Griebel, Multiple intestinal 'loops' provide an in vivo model to analyse multiple mucosal immune responses, J. Immunol. Methods 256 (2001) 19-33.
[19] S.Y. Seong, N.H. Cho, I.C. Kwon, S.Y. Jeong, Protective immunity of microsphere-based mucosal vaccines against lethal intranasal challenge with Streptococcus pneumoniae, Infect. Immun. 67 (1999) 3587-3592.

[20] B. Kim, T. Bowersock, P. Griebel, A. Kidane, L.A. Babiuk, M. Sanchez, S. Attah-Poku, R.S. Kaushik, G.K. Mutwiri, Mucosal immune responses following oral immunization with rotavirus antigens encapsulated in alginate microspheres, J. Control. Release 85 (2002) 191-202.

[21] V. Dodane, M. Amin Khan, J.R. Merwin, Effect of chitosan on epithelial permeability and structure, Int. J. Pharm. 182 (1999) 21-32.

[22] G.D. Guerra, P. Cerrai, M. Tricoli, S. Maltinti, R.S. del Guerra, In vitro cytotoxicity testing of chitosan-containing polyelectrolyte complexes, J. Mater. Sci., Mater. Med. 9 (1998) 73-76.

[23] B.I. Florea, M. Thanou, H.E. Junginger, G. Borchard, Enhancement of bronchial octreotide absorption by chitosan and $\mathrm{N}$-trimethyl chitosan shows linear in vitro/in vivo correlation, J. Control. Release 110 (2006) 353-361.

[24] Z. Shi, K.G. Neoh, E.T. Kang, W. Wang, Antibacterial and mechanical properties of bone cement impregnated with chitosan nanoparticles, Biomaterials 27 (2006) 2440-2449.

[25] G. Orive, A.M. Carcaboso, R.M. Hernandez, A.R. Gascon, J.L. Pedraz, Biocompatibility evaluation of different alginates and alginate-based microcapsules, Biomacromolecules 6 (2005) 927-931.

[26] K.J. Tønnesen, Alginate in Drug Delivery Systems, Drug Dev. Ind. Pharm. 28 (2002) 621-630.

[27] P. de Vos, C.G. Hoogmoed, H.J. Busscher, Chemistry and biocompatibility of alginate-PLL capsules for immunoprotection of mammalian cells, J. Biomed. Mater. Res. 60 (2002) 252-259.

[28] N.H. Cho, S.Y. Seong, K.H. Chun, Y.H. Kim, I.C. Kwon, B.Y. Ahn, S.Y. Jeong, Novel mucosal immunization with polysaccharide-protein conjugates entrapped in alginate microspheres, J. Control. Release 53 (1998) 215-224.

[29] F.O. Costa, J.J. Sousa, A.A. Pais, S.J. Formosinho, Comparison of dissolution profiles of Ibuprofen pellets, J. Control. Release 89 (2003) 199-212.

[30] F.L. Mi, S.S. Shyu, C.T. Chen, J.Y. Schoung, Porous chitosan microsphere for controlling the antigen release of Newcastle disease vaccine: preparation of antigen-adsorbed microsphere and in vitro release, Biomaterials 20 (1999) $1603-1612$.

[31] J. Benesch, P. Tengvall, Blood protein adsorption onto chitosan, Biomaterials 23 (2002) 2561-2568.

[32] B. Magenheim, S. Benita, Nanoparticle characterization: a comprehensive physicochemical approach, S. T. P. Pharma Sci. 4 (1991) 221-241.

[33] Y. Xu, Y. Du, Effect of molecular structure of chitosan on protein delivery properties of chitosan nanoparticles, Int. J. Pharm. 250 (2003) 215-226.

[34] I.M. van der Lubben, J.C. Verhoef, A.C. van Aelst, G. Borchard, H.E. Junginger, Chitosan microparticles for oral vaccination: preparation, characterization and preliminary in vivo uptake studies in murine Peyer's patches, Biomaterials 22 (2001) 687-694.

[35] I.M. van der Lubben, G. Kersten, M.M. Fretz, C. Beuvery, J. Coos Verhoef, H.E. Junginger, Chitosan microparticles for mucosal vaccination against diphtheria: oral and nasal efficacy studies in mice, Vaccine 21 (2003) 1400-1408.

[36] E.Y. Chi, S. Krishnan, T.W. Randolph, J.F. Carpenter, Physical stability of proteins in aqueous solution: mechanism and driving forces in nonnative protein aggregation, Pharm. Res. 20 (2003) 1325-1336.

[37] C. Desert, C. Guerin-Dubiard, F. Nau, G. Jan, F. Val, J. Mallard, Comparison of different electrophoretic separations of hen egg white proteins, J. Agric. Food Chem. 49 (2001) 4553-4561.

[38] C. Washington, Drug release from microparticulate systems, in Microencapsulation: Methods and Industrial Applications, in: S. Benita (Ed.), Marcel Dekker - Taylor and Francis CRC, 1996, pp. 155-181.

[39] K. Roy, H.Q. Mao, S.K. Huang, K.W. Leong, Oral gene delivery with chitosan-DNA nanoparticles generates immunologic protection in a murine model of peanut allergy, Nat. Med. 5 (1999) 387-391.

[40] A. Berthold, K. Cremer, J. Kreuter, Preparation and characterization of chitosan microspheres as drug carrier for prednisolone sodium phosphate as model for anti-inflammatory drugs, J. Control. Release 39 (1996) 17-25.

[41] R. Hejazi, M. Amiji, Stomach-specific anti-H. pylori therapy. I: Preparation and characterization of tetracyline-loaded chitosan microspheres, Int. J. Pharm. 235 (2002) 87-94. 
[42] R. Fernandez-Urrusuno, P. Calvo, C. Remunan-Lopez, J.L. Vila-Jato, M.J. Alonso Enhancement of nasal absorption of insulin using chitosan nanoparticles, Pharm. Res. 16 (1999) 1576-1581.

[43] A. Vila, A. Sanchez, K. Janes, I. Behrens, T. Kissel, J.L. Vila Jato, M.J. Alonso, Low molecular weight chitosan nanoparticles as new carriers for nasal vaccine delivery in mice, Eur. J. Pharm. Biopharm. 57 (2004) 123-131.

[44] M.K. Lai, C.Y. Chang, Y.W. Lien, R.C. Tsiang, Application of gold nanoparticles to microencapsulation of thioridazine, J. Control. Release 111 (2006) 352-361.

[45] N.V. Mulye, S.J. Turco, A simple model based on first order kinetics to explain release of highly water soluble drugs from porous dicalcium phosphate dihydrate matrices, Drug Dev. Ind. Pharm. 21 (1995) 943-953.

[46] C.J. Hitzman, T.S. Wiedmann, H. Dai, W.F. Elmquist, Measurement of drug release from microcarriers by microdialysis, J. Pharm. Sci. 94 (2005) $1456-1466$.

[47] J.E. Eyles, V.W. Bramwell, J. Singh, E.D. Williamson, H.O. Alpar, Stimulation of spleen cells in vitro by nanospheric particles containing antigen, J. Control. Release 86 (2003) 25-32.
[48] R.E. Mebius, G. Kraal, Structure and function of the spleen, Nat. Rev., Immunol. 5 (2005) 606-616.

[49] M. Amidi, S.G. Romeijn, G. Borchard, H.E. Junginger, W.E. Hennink, W Jiskoot, Preparation and characterization of protein-loaded N-trimethyl chitosan nanoparticles as nasal delivery system, J. Control. Release 111 (2006) 107-116.

[50] V.K. Shreedhar, B.L. Kelsall, M.R. Neutra, Cholera toxin induces migration of dendritic cells from the subepithelial dome region to T- and B-cell areas of Peyer's patches, Infect. Immun. 71 (2003) 504-509.

[51] A. Kidane, P. Guimond, T.C. Rob Ju, M. Sanchez, J. Gibson, A. North, H. HogenEsch, T.L. Bowersock, Effects of cellulose derivatives and poly (ethylene oxide)-poly(propylene oxide) tri-block copolymers (Pluronic) surfactants on the properties of alginate based microspheres and their interactions with phagocytic cells, J. Control. Release 85 (2002) 181-189. 